\title{
Intervención Social con la Niñez: operacionalizando el enfoque de derechos
}

FRANCIS VALVERDE MOSQUERA

Postítulo en Evaluación y Currículo, PUC. Experta en Derechos Humanos, Universidad Prince Edward Island, Canadá. Experta en elaboración y evaluación de proyectos, SUR-CEPAL. Docente de Cátedra Universidad Academia Humanismo Cristiano. Coordinadora Ejecutiva ACHNU francisvalverde@gmail.com

\section{Introducción}

El discurso sobre los derechos de niños y niñas ${ }^{[1]}$ a partir de la promulgación de la Convención sobre los Derechos del Niño (CDN), se ha hecho común entre los y las profesionales que se relacionan con el tema, ya sea del sector público como de las instituciones que basan su actuar en ella. De manera similar han incorporado el hablar de enfoque de derechos. No ocurre lo mismo con muchos de estos profesionales que trabajando con la infancia y la niñez, todavía tienen un enfoque asistencial de la intervención social, y visualizan al niño y la niña como receptores de servicios, y no como sujetos de derechos, más allá que puedan en sus escritos y documentos utilizar las expresiones derechos del niño y sujeto de derechos. El desafío, por tanto, consiste en poner en práctica y operacionalizar estos discursos en las intervenciones directas con niños y niñas, en trabajar con ellos y ellas, haciendo de la intervención social un espacio de desarrollo personal y colectivo, un

[1] En este artículo se usará permanentemente los conceptos niños y niñas, pues a diferencia del idioma inglés, en el idioma español no existe un concepto que incluya ambos. Es relevante que cuando se lea el texto se incluya la diferencia de género, ya que desde la imagen social como en el ejercicio de derechos existe una diferencia de género que tiene que hacerse explícita.

Esto es tan relevante, que una de las reformas constitucionales más debatidas y de difícil alcance fue la reforma del artículo 19: La Constitución asegura a todas las personas: (...); que en su inciso $1^{\circ}$ cambió la palabra hombre por persona, concepto asexuado, quedando: Artículo $1^{\circ}$. Las personas nacen libres e iguales en dignidad y derechos; y en su inciso $2^{\circ}$ agregó la palabra mujeres, quedando: La igualdad ante la ley. En Chile no hay persona ni grupo privilegiados. En Chile no hay esclavos y el que pise su territorio queda libre. Hombres y mujeres son iguales ante la ley. Constitución Política de la República de Chile de 1980, reforma de 1999. 
espacio de ejercicio de derechos y de práctica ciudadana. Esto obliga a los adultos a establecer relaciones de colaboración, respeto e igualdad con las niñas y los niños, en tanto ambos son sujetos de derechos.

\section{Conceptualizando}

El enfoque de derechos empieza a ser utilizado como un concepto desde fines de los años noventa del siglo XX, para dar cuenta del grado de involucramiento que tenían las políticas públicas dirigidas a la infancia, niñez y adolescencia, con los principios planteados por la Convención sobre los Derechos del Niño (CDN). Estas políticas hacen una enunciación de los derechos correspondientes pero no dan cuenta de las implicancias prácticas que estos derechos tienen en la implementación de ellas.

A principios de la década actual, la alianza Save the Children entrega el primer acercamiento a una "programación de los derechos del niño" (PDN), que en definitiva establece los fundamentos de lo que hoy entendemos por enfoque de derechos, cuando de niñas y niños se trata.

Este enfoque tiene dos ejes rectores. El primero, una concepción de desarrollo, y el segundo, los derechos humanos como base de una propuesta de implementación concreta de estos derechos en la vida cotidiana de niñas, niños y adolescentes.

El primer eje mencionado da cuenta de la relevancia que tienen los derechos humanos en las políticas de desarrollo, tanto de los países como de las personas, y que han sido utilizados como fundamento de las mismas, como un ideal a alcanzar desde la Segunda Guerra Mundial. Amartya Sen ${ }^{[2]}$ hace una definición de desarrollo que incluye uno de los elementos constitutivos de los DDHH, a saber, la libertad. "El desarrollo puede concebirse..., como un proceso de expansión de las libertades reales de que disfrutan los individuos" y continúa, "El desarrollo exige la eliminación de las principales fuentes de privación de libertad: la pobreza, la tiranía, la escasez de oportunidades económicas y las privaciones sociales sistemáticas, el abandono en que

[2] Sen, Amartya. Desarrollo y Libertad, Editorial Planeta, Barcelona, 2000. Pág. 19. 
puedan encontrarse los servicios públicos y la intolerancia o el exceso de intervención de los Estados represivos". ${ }^{[3]}$

Lo anterior está en conjunción con los desafíos que se han planteado los países en las últimas décadas, donde los gobiernos se han puesto de acuerdo en el cumplimiento de los derechos humanos como un objetivo de desarrollo, el que ha quedado plasmado en la Declaración del Milenio de Naciones Unidas y los correspondientes objetivos de desarrollo del milenio. ${ }^{[4]}$

Esta definición de desarrollo de Sen, puede ser complementada con la utilizada por Francisco de Oliveira ${ }^{[5]}$, quien lo entiende "como un proceso sustentado de crecimiento económico con distribución de riqueza y renta. (...) El proceso de desarrollo debe ser procesado de forma democrática, es decir, con la activa participación e intervención de las clases sociales en la distribución del excedente económico".

El desarrollo entendido en estos dos aspectos, a saber, la expansión de las libertades por una parte, y el crecimiento económico con distribución efectiva de riqueza basado en un proceso democrático y participativo, por otro, pone en evidencia que una sin el otro no son posibles. No es posible la expansión de las libertades en un régimen político, social cultural y económico excluyente, que impide la realización de las potencialidades humanas. Este dato es relevante cuando las estadísticas en América Latina nos muestran que la infancia y la niñez son parte fundamental de los altos porcentajes de pobreza de cada país.

El segundo eje, referido a los Derechos Humanos y sus principios, parte con la afirmación de que los derechos del niño establecidos en la CDN son derechos humanos. Esto que pareciera una redundancia, no lo es cuando se observa que en la sociedad, las niñas y los niños siguen siendo personas de

[3] El segundo considerando de la Declaración Universal de Derechos Humanos de 1948, establece como "la aspiración más elevada del hombre el advenimiento de un mundo en que los seres humanos, liberados del temor y de la miseria, disfruten de la libertad de palabra y de la libertad de creencias".

[4] Véase la Declaración del Milenio de Naciones Unidas en www.un.org/ milleniumdeclaration/ares552e.htm , y los Objetivos del Desarrollo para el Milenio en www.developmentgoal.org

[5] De Oliveira, Francisco. "Sobre las posibilidades del desarrollo", en: América Latina 2004-2005. Democracia y Desarrollo: Una mirada desde la sociedad civil. Informe de ALOP, Asociación latinoamericana de Organizaciones de Promoción. 2005. Pág. 45. 
segunda categoría, invisibilizados y no asumidos como sujetos con derechos al igual que los adultos. La CDN establece los derechos civiles, sociales y culturales muy claramente, no así los derechos políticos, que corresponden a los de representación, a la posibilidad de elegir y ser elegidos, de ser considerados en la toma de decisiones políticas de las sociedades de las cuales son parte. Como dice Lourdes Gaitán ${ }^{[6]}$, "Los niños pueden entenderse como componentes de grupo minoritario, caracterizado por encontrarse todos sus miembros por debajo de una edad. La condición de minoría social comporta una discriminación en materia de derechos, acceso al poder, bienestar y prestigio, así como una subordinación al grupo dominante".

\section{Niños y niñas como sujetos sociales}

Cuando se habla del niño y la niña como sujeto, estamos entrando de lleno a la comprensión del mismo como sujeto social, como parte de un colectivo y de un grupo social. Deja de ser visto de manera individual caracterizado por la psicología y la pedagogía como un individuo en preparación para, inmaduro, incompleto, incapaz, etc. ligado por tanto casi exclusivamente a su proceso de desarrollo. Tal como lo plantea Touraine, en la modernidad surge el" sujeto humano como libertad y creación" ${ }^{[7]}$ En otras palabras es el individuo capaz de asumir su capacidad de transformación de la realidad, y por tanto de constituirse en actor social. ${ }^{[8]}$ En definitiva, la idea de sujeto es la de un resistente y combatiente por la libertad. ${ }^{[9]}$

Asumir a niñas y niños como sujetos sociales, entendidos estos en tanto la conjunción del individuo, sujeto y actor, donde cada uno se define en relación a los otros dos ${ }^{[10]}$, en un proceso que como dice Touraine, se resiste al individualismo que solo lo reduce a la razón, y más bien releva el hecho de que en el sujeto se asocia individuo y libertad. Entender a niños y niñas como

[6] Gaitán, Lourdes. Sociología de la Infancia, Nuevas Perspectivas, Madrid, 2006, Pág. 20.

[7] Touraine Alain, Crítica de la Modernidad, Fondo de Cultura Económica, Argentina, 1994, Pág. 205

[8] Op.cit pág. 207

[9] Touraine Alain, Un nuevo paradigma: para comprender el mundo de hoy, Editorial Paidós, 2006, Pág. 130

[10] Touraine Alain, Crítica a la Modernidad (...) Op.cit Pág. 208 
sujetos sociales implica que estos pasan a ser parte constitutiva del tejido social y cultural del cual forman parte, por ende se les asume como sujetos activos, públicos, parte de un colectivo. De esta manera deben dejar de ser vistos como víctimas indefensas del destino, constituyéndose en sujetos capaces de proponer soluciones a las situaciones de vulneración en que viven, donde pueden y deben tomar parte en la toma de decisiones que les afectan a ellos y a la comunidad ${ }^{[11]}$ en la cual viven. Así planteado se constituyen en actores sociales, en ciudadanos. Esto evidentemente, cuestiona las bases mismas del sistema de relaciones de poder que se dan entre adultos y niños y obliga a repensar las formas de participación de cada uno de los actores sociales, siendo uno de ellos, las niñas y los niños, o la infancia y la niñez.

\section{Enfoque en intervención social}

Volviendo a la noción de enfoque, este lo estamos entendiendo como una manera particular de observar, valorar en involucrarse con la realidad en una temática específica, en este caso, la realidad de niños y niñas en nuestra sociedad.

Es importante tener presente que no hay enfoques o formas de mirar la realidad político-social neutros. Todo enfoque tiene en su base una ideología que lo sustenta, entendida esta básicamente como un conjunto de principios y directrices que le dan una comprensión del mundo; tienen una determinada valoración de los sujetos; un sentido, una proposición de hacia donde ir, lo que aspiran alcanzar (lo ideal), y una modalidad de intervención, ${ }^{[12]}$ cuando de realidades sociales se trata, que trataremos más adelante.

Entonces, podemos decir que el enfoque de derechos es un enfoque de desarrollo que nos obliga a mirar la realidad desde el relevamiento de la responsabilidad del Estado, en tanto garante principal de derechos; la certeza de que los sujetos de derechos pueden y deben exigir / reclamar sus derechos; la existencia de obligaciones legales por parte de los garantes, expresadas en los instrumentos internacionales de derechos humanos; y el reconocimiento del niño y la niña como sujeto y actor social.

[11] Entenderemos comunidad en este artículo como el grupo social inmediato en que se desarrollan los niños y las niñas, y que será objeto de una intervención social.

[12] Carballeda J. Alfredo, La intervención en lo social. Exclusión e integración en los nuevos escenarios sociales, Serie Tramas Sociales, Editorial Paidós, Buenos Aires, 2005. 
Lo relevante del Enfoque de Derechos es que se fundamenta en un conjunto de valores y principios sobre los seres humanos y sobre el desarrollo expresados a través de los derechos humanos.

El enfoque de derechos, es en definitiva un conjunto de propuestas, que se hacen efectivas a través de su aplicación sistemática y completa, teniendo una clara visión de los modos de intervención que se han de utilizar, el rol que cumplirán en ellas los niños y las niñas, las formas de medir los logros alcanzados, y la sustentabilidad de los cambios buscados.

Cuando se trabaja con este enfoque, a lo menos en términos teóricos, se tienen una serie de beneficios, siendo algunos de ellos:

- El acuerdo internacional y legitimidad, expresados en un marco jurídico internacional que comparten los gobiernos y la sociedad civil. Este marco se puede traducir en estándares acordados por la mayoría de los países del mundo.

- Un objetivo de largo plazo: el cumplimiento de los derechos humanos, el cual puede ir siendo medido a medida que se avanza hacia su logro.

- La exigibilidad de la rendición de cuentas, que expresan claramente las responsabilidades de los gobiernos, y de todos los actores que intervienen en los cambios societales.

- El proceso de empoderamiento, entendido como el proceso de asunción de sus propias capacidades, habilidades y potencialidades que viven las personas y que se requiere para el pleno ejercicio de derechos. En otras palabras, lograr la participación activa de los grupos menos favorecidos y más vulnerados en las sociedades, para alcanzar justicia social, no-discriminación y desarrollo de los sectores pobres, es decir, inclusión y no marginación.

- El objetivo de alcanzar la equidad, para lo cual se trabaja intencionadamente por la justicia, la igualdad y la libertad, principios fundantes de los derechos humanos, lo que obliga a enfrentar problemas de poder que están a la base de la inequidad, la pobreza y la explotación. 
- Un mayor impacto y eficacia, debido al énfasis que pone en la rendición de cuentas, el pleno ejercicio de derechos y la participación activa de los distintos actores sociales.

- Un enfoque holístico, que permite trabajar sobre la integralidad de la cuestión a enfrentar y no parcelizar y/o atomizar ya sea la realidad o a las personas que la viven. ${ }^{[13]}$

Hasta aquí lo relacionado con enfoque de derechos que ampliaremos más adelante cuando lo interrelacionemos con intervención social. Ahora desarrollaremos como entenderemos la intervención social con enfoque de derechos en este artículo.

La intervención social, puede entenderse como una irrupción en una realidad determinada con la intención de modificarla. Alude a un actuar desde "fuera", a la acción de un agente externo que se "introduce" en esa realidad de la cual no es parte.

Es importante tener presente que toda intervención social, tiene una hipótesis de acción que la fundamenta y le da sentido, así como evidencia la propuesta de cambio que tiene, la cual en la mayoría de los casos no está explícita. La hipótesis involucra una evaluación de la situación inicial sobre la cual se quiere intervenir, un proceso de construcción de comunidad y una anticipación de una situación alternativa posible.

[13] Tomado de Programación de los Derechos del Niño. Cómo aplicar un Enfoque de Derechos en la programación. Un manual para los miembros de la Alianza Internacional Save the Children. Segunda Edición. Lima - Perú 2005. 


\section{Hipótesis de Acción ${ }^{[14]}$}

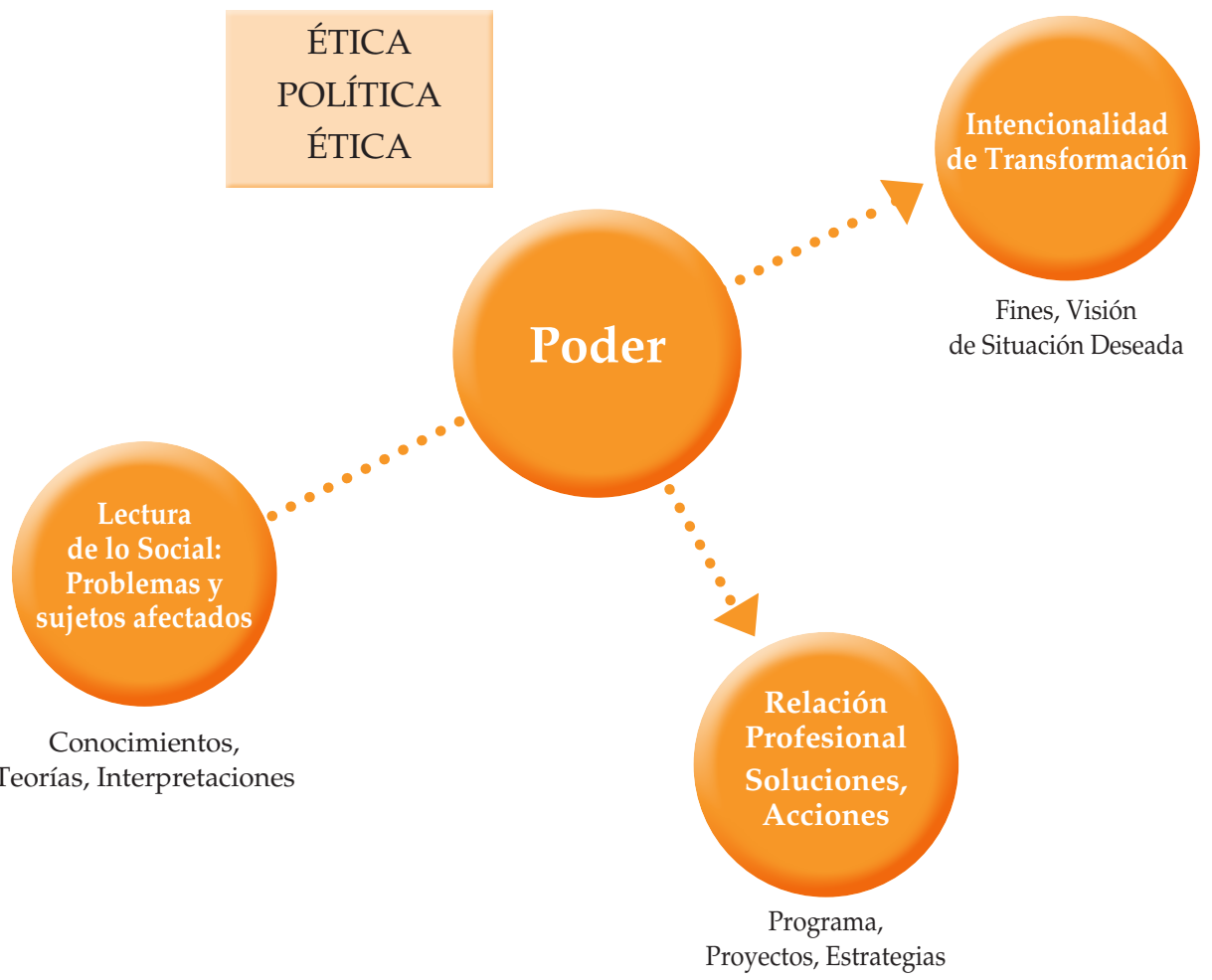

El diagrama da cuenta de lo dicho anteriormente. La lectura de lo social es un diagnóstico e interpretación de la sociedad en la que se vive, la que se fundamenta en teorías, modelos explicativos, ideologías, etc., que dan cuenta de una situación inicial que releva los problemas y los sujetos afectados por ellos, y aproxima una metodología de cómo enfrentarlo. El modo de concebir lo social conlleva una forma de comprender las situaciones o "problemas sociales" y los sujetos afectados, incidiendo en los modos de vinculación y acción frente a éstos.

[14] Cuadro tomado de presentación en transparencias del Módulo Intervención Social y Enfoque de Derechos, de las docentes Soledad Latorre y Francis Valverde, del Diplomado “Niñez y Políticas Públicas” U. de Chile 2007. 
Los denominados "problemas sociales" se relacionan generalmente con derechos humanos no cumplidos, necesidades insatisfechas, demandas de intervención, descontento, malestar frente a condiciones poco humanas, desigualdad, desventaja o exclusión, entre otros.

Los problemas sociales que afectan a las personas de una comunidad determinada, denominados sujetos de intervención, son una "construcción" de los interventores, generalmente profesionales, que responden a políticas sociales elaboradas desde un enfoque político y social determinado, y que tiene sobre los intervenidos una valoración, ya sea de afectado, víctima, beneficiario, protagonista o participante, lo que hará una diferencia fundamental al establecer el vínculo relacional con ellos y ellas, en el momento de la intervención, y por lo tanto de los resultados de la misma.

El otro elemento importante de tratar en esta sección con respecto a la Hipótesis de Acción, dice relación con el Poder, concepto eminentemente político, que desde los derechos humanos está relacionado con los derechos civiles y políticos y que generalmente cuando está referido a la intervención social de niños y niñas, se deja fuera, asumiendo erróneamente, que ellos y ellas no tienen o no pueden ejercer estos derechos, por lo tanto, se pone especial énfasis en los aspectos sociales y culturales de la intervención, dejando de lado toda la reflexión sobre el poder, tanto de los interventores como el de los intervenidos.

Es relevante tener presente que toda intervención se constituye en un espacio político, en el que se establecen relaciones de poder y donde hay una búsqueda de influir sobre la voluntad del otro. Hay una relación asimétrica entre los profesionales que intervienen y los sujetos de intervención; existe un poder dado a estos profesionales por su conocimiento, status, prestigio establecido dentro de un contexto institucional determinado.

Es a partir de esta noción de poder, de la ideología a la base de la propuesta de intervención, donde aparece o tendría que aparecer la intencionalidad transformadora, el cambio social deseable, que debiera ser en definitiva el resultado de la intervención y que se expresa en una apuesta en los cambios de condiciones de vida de los sujetos, en una aspiración de tipo de sociedad por la que se apuesta. En otras palabras, existe una definición de lo deseable, legítimo, aceptable y lo posible, todos elementos que orientan el tipo de cambio esperado.

Lo que debe tenerse presente es que no hay una intervención social neutra ni espontánea, toda intervención se sustenta en una teoría de cambio, 
la que tiene una fundamentación política, ideológica y axiológica. El desafío consiste en explicitar esta teoría de cambio durante el desarrollo de la intervención, no solo a los y las profesionales que la están realizando, sino y fundamentalmente a los sujetos afectados por esa intervención.

Finalmente, y asumiendo la realidad político social actual, se puede afirmar que la intervención social hoy tiene como uno de sus objetivos fundamentales la resolución de la problemática de la integración social ${ }^{[15]}$, esto considerando la tensión inclusión/exclusión existente. Aquí los supuestos que están a la base serían la fragmentación social, la segregación territorial, el no cumplimiento ni ejercicio pleno de derechos por parte de la ciudadanía, de la cual forman parte los niños, niñas y adolescentes.

\section{Intervención Social desde un Enfoque de Derechos}

Una vez explicitados los conceptos básicos de trabajo, tres elementos sustantivos hay que tener en cuenta para pensar y actuar la intervención social desde el enfoque de derechos.

El primer elemento sustantivo es que la intervención social ha de ser entendida como un espacio de desarrollo y relevamiento de las capacidades, potencialidades y habilidades individuales y colectivas de niñas, niños y adolescentes en tanto actores sociales; el segundo, el niño y la niña han de ser tratados como "partícipes" y no como beneficiarios de la intervención social; y el tercero, establecer el rol de garantes de derechos de los intervinientes o interventores, sean estos organizaciones de la sociedad civil o agencias del Estado.

Si recordamos que la intervención social es un dispositivo, al cual subyace una Hipótesis de Acción, que involucra una evaluación de una situación inicial, un proceso de construcción de comunidad y una anticipación de una situación alternativa posible, entonces es imprescindible preguntarse cuál es la situación inicial sobre la cual queremos intervenir.

Esta situación inicial vista desde el enfoque de derechos, está marcada por el no cumplimiento ni ejercicio de derechos de las personas en general, en nuestro caso específico, los de las niñas y niños. Situar la mirada en el no

[15] Carballeda J. Alfredo, op.cit. 
ejercicio de derechos de niñas y niños obliga a pensar que ellos y ellas tienen la capacidad y posibilidad de ejercer esos derechos pero que no tienen la oportunidad ni las condiciones para ejercerlos, asumiendo que ellos y ellas los conocerían y sentirían propios.

Aparece entonces fundamental establecer qué es lo que se está entendiendo por derechos humanos, pues eso nos permitirá marcar toda la intervención social. Los derechos humanos y cada derecho humano lo entenderemos, en palabras de Domínguez "como un patrón de relación social, apto a la libre y original forma de realizarse que habita al ser humano y que la autoridad debe promover, defender y ayudar a realizar..."[16] En otras palabras los derechos humanos son formas de relación social, política, económica y cultural, que regulan los vínculos entre las personas y los Estados, en la lógica de establecerle tanto un límite al poder del Estado frente a los ciudadanos, como una obligación de éste de facilitar el ejercicio de los derechos a todos y todas sin discriminación alguna (derechos civiles y políticos); además de obligarle a relacionarse con la ciudadanía de manera de asegurar la dignidad en el desarrollo de cada persona creando las condiciones que permitan el desarrollo pleno de todas las personas (derechos económicos, sociales y culturales). Así planteado los Derechos Humanos han de ser pensados"como relaciones de justicia y solidaridad que abren los espacios para la libre determinación creadora de las personas, grupos y pueblos a los cuales están obligados todos los Estados."[17]

Asumida esta noción de los derechos humanos, relevar cual es el real conocimiento y capacidad de ejercicio de derechos de niñas y niños, será la primera acción intencionada de una intervención social, tomando en cuenta primero, que no solo para los niños y niñas los derechos son de corta data, sino que hay toda una historia donde los derechos de ellas y ellos no eran parte ni siquiera del discurso de los adultos. Segundo, que la aparición formal de los derechos de niños y niñas, no los hace automáticamente parte de la cultura relacional de ellos y de los adultos con ellos y ellas; la cultura asume hasta hoy la relación de dependencia y sujeción de los menores de edad a los mayores de edad. Tercero, la no comprensión en general de qué son los

[16] Domínguez V. Andrés, El poder y los Derechos Humanos, CEPLA, Terranova Editores, 1988, Pág. 15

[17] Domínguez V. Andrés, op.cit. Pág. 14 
derechos humanos y cómo éstos nos llevan a pensar y actuar en las relaciones entre las personas desde otros parámetros, valores y sentidos.

La intervención social desde el enfoque de derechos implica que la construcción de la idea sobre la situación inicial, que generalmente el equipo interventor tiene medianamente clara, ha de ser contrastada con la que dicen tener y vivir los intervenidos, en este caso la comunidad y los niños y niñas. Por lo tanto, el rol de los profesionales será el de facilitadores en la comprensión de la realidad que se quiere intervenir con la comunidad, creando un vínculo de respeto, confianza y apertura, generando espacios de diálogo y reflexión conjunta, y aceptando los límites que la comunidad pone a la intervención, en la perspectiva que la hagan suya, la sientan propia y necesaria y por lo mismo se comprometan con los cambios buscados.

Las estrategias metodológicas para alcanzar esto son variadas y dependerán en gran medida del grado de conocimiento que la comunidad y los niños y niñas tengan de sus derechos y de cuales son las necesidades a satisfacer. Esto está íntimamente ligado al grado de cohesión social que tengan en tanto comunidad; al nivel de desarrollo organizacional existente tanto entre los adultos como entre los niños y niñas; a la capacidad que han tenido de hacerse escuchar por las autoridades y en el caso de niñas y niños por los adultos (capacidad de incidencia); a las otras intervenciones que está viviendo la comunidad y los sentidos de éstas y cuán complementarias son con las que se están proponiendo; a la memoria social y política de las luchas de esa comunidad que le permitan construir su propia historia donde prevalezcan sus aprendizajes y sus logros.

Así planteado, el desafío del equipo profesional interviniente es muy alto pues tiene que tener un conocimiento previo de la situación e historia de esa comunidad y de cómo los niños y las niñas la viven previa a la llegada a la misma, de manera de asegurar que no "irrumpirán" en un realidad imponiendo acciones que no dicen relación con la historia y necesidades sentidas por ellos y ellas. El equipo profesional que interviene, desde un enfoque de derechos requiere de un estudio y conocimiento previo lo más detallado posible de la situación de la comunidad y de las niñas y niños dentro de ella, de manera que el período de instalación de la intervención, es decir, el momento desde que llegan hasta el momento en que la comunidad los acepta y asume como válidos (si no propios), sea lo más breve posible, de manera que los alcances de la intervención o el cambio esperado, se logre en los tiempos requeridos por la comunidad. 
Por ello, es que la intervención ha de constituirse en un espacio de desarrollo y empoderamiento colectivo de niñas, niños y adolescentes en tanto actores sociales, pues es en el proceso de instalación, donde la creación de un vínculo expedito, fluido, respetuoso y pertinente culturalmente del equipo profesional con ellos y ellas marcará toda la intervención. En este proceso niñas, niños y adolescentes se asumen importantes para el éxito de la intervención al sentirla propia, al ser parte de la reflexión respecto de la situación que están viviendo y que quieren cambiar, al construir junto con el equipo alternativas de solución y de cambio de la situación inicial en la cual asumen un rol concreto y relevante; ellas y ellos tienen ideas y las pueden llevar a la práctica, así asumen responsabilidad en mejorar las condiciones de vida de su comunidad, que desde el enfoque de derechos, es la generación de condiciones de ejercicio de los derechos para todos y todas las personas de la comunidad, no solo de las niñas y los niños.

El proceso a través del cual niñas, niños y adolescentes se van constituyendo en actores sociales, dice relación con el proceso que ellos y ellas viven cuando van asumiendo que tienen capacidades para actuar en la realidad, que tienen ideas y que estas son válidas y pertinentes a las necesidades que visualizan, que pueden actuar en conjunto con otros de manera colectiva, lo que les da fuerza y autonomía en la acción, etc. Este proceso no es fácil ni rápido, de hecho es un proceso con altos y bajos, con rupturas y dificultades la mayor parte de las veces puestas por los adultos, quienes no entienden esta lógica de los derechos de niñas y niños, y los ven más como un desacato a su autoridad, a lo que ellos consideran debe hacerse. Se rompe la relación preestablecida de "obediencia debida" de niñas y niños hacia los adultos y esto por supuesto inseguriza a los adultos y a las autoridades, por lo cual intentarán limitar lo más posible esta autonomía colectiva que se está gestando en ellos y ellas.

En este proceso la tarea del equipo interventor es ardua, pues en gran medida tiene que hacer de mediador entre los niños, las niñas y los adultos, a la vez que trabajar en la formación tanto de niñas y niños como de los adultos para que esta nueva forma de relación entre ellos sea asumida de manera positiva y fructífera. Estamos hablando ni más ni menos de aportar en el cambio de las relaciones de poder que hasta el momento han sido asumidas

[18] Un ejemplo de esto es la reciente situación vivida por la estudiante María Música con la Ministra de Educación Mónica Jiménez. 
como las únicas válidas y correctas, que son de obediencia, sumisión y aceptación de lo dicho y hecho por el adulto. ${ }^{[18]}$ El enfoque de derechos no hace más que poner en evidencia que esta forma de relación no corresponde a la que debe tenerse entre sujetos de derechos iguales en dignidad y derechos pero con distintas responsabilidades. El cambio esperado con este proceso no se alcanza en una ni en varias intervenciones, lo que hace cada intervención con enfoque de derechos es iniciar los procesos para que este cambio de más largo aliento, que es de tipo cultural, pueda ocurrir. Por lo anterior, un desafío fundamental y que tiene que tener claro el equipo interventor, son las estrategias de participación de niñas, niños y adolescentes en cada intervención y las formas cómo éstas se complementan con la de los adultos.

En este artículo se ha puesto especial énfasis a la fase de instalación de una intervención social con enfoque de derechos, toda vez que ésta marcará toda la actividad posterior, fase en la cual adquiere especial relevancia la evaluación de la situación inicial, la cual debe ser construida en conjunto con la comunidad y con los niños, las niñas y los adolescentes en particular.

No hay que olvidar, que toda intervención social con enfoque de derechos tiene además una imagen clara del proceso de construcción de comunidad y la situación deseada al término de la misma. Al igual que con la situación inicial, tanto el proceso como la situación final deseada han de ser reflexionadas, discutidas, dialogadas y consensuadas con la comunidad. Muchos de los conceptos y prejuicios con los que se inicia la intervención serán cambiados y / o cuestionados durante el proceso mismo. Por lo anterior es importante que el equipo profesional sea flexible, abierto y esté dispuesto a aceptar las propuestas que vienen de las niñas y niños de la comunidad, pues su rol fundamental es aportar al proceso de empoderamiento de ellos y ellas y de la comunidad toda, asumiendo la responsabilidad profesional de aportar sus conocimientos para que el proceso se pueda dar. Por ello deben ser capaces de mediar, formar, capacitar, generar estrategias metodológicas variadas y sistematizar la experiencia, de manera que el aprendizaje a partir de la práctica sea un bien utilizado por la comunidad y por otros profesionales.

Son estos procesos en conjunto los que permiten decir que la intervención social, desde el enfoque de derechos, se constituye en un espacio de empoderamiento y desarrollo colectivo de niñas, niños y adolescentes con la comunidad y, por que no, del equipo profesional que interviene en esa realidad. No hay que olvidar que son los procesos y cómo estos se desarrollan, los que producen los cambios social, cultural y políticamente sustentables 
El segundo elemento sustantivo de una intervención social con enfoque de derechos dice relación con la forma del vínculo que se establece con las niñas, los niños y adolescentes que son parte de ella. En general los equipos que intervienen socialmente responden a las ideas y prejuicios que están a la base de sus apuestas de cambio, y por lo tanto, éstas conllevan una valoración de los "intervenidos" que determina la forma de relación que establecerán con ellos, y por lo tanto, la forma en como éstos serán parte de las opciones asumidas de intervención que se estén implementando.

Lo primero, que hay que tener claro es que cuando nos referimos a niñas y niños como sujetos de derecho, estamos afirmando que son personas que tienen todas las capacidades para ejercer sus derechos acorde a la edad que tienen, es decir, son sujetos plenos, interlocutores válidos y fundamentales en una intervención social que pretende satisfacer sus necesidades o que está estructurada para ellos y ellas. Esto implica cuestionarse las formas en que valoramos por una parte la forma de relación que tenemos con ellas y ellos, y por otra, la manera en que visualizamos y estructuramos su actuar en la propuesta de intervención.

La inclusión de niñas y niños como sujetos de derecho, en términos formales y universales recién ocurre con la promulgación de la Convención sobre los Derechos del Niño de 1989. La Convención marca un antes y un después en la valoración social de niñas y niños e inicia un fuerte proceso de reflexión de las formas en que la infancia, la niñez y la adolescencia son comprendidas, y las nuevas implicancias que esto trae para efectos de políticas públicas.

Este hecho en si mismo se constituye en una acción transformadora e incluso peligrosa, cuando viene de aquellas personas que se consideraban en preparación para, con una cantidad de incapacidades, lo que nos obligaba a protegerlos para que crecieran y llegaran a ser los adultos responsables y adecuados que la sociedad necesitaba.

Se podría decir que la gran dificultad para trabajar los derechos del niño, o realizar una intervención social desde el enfoque de derechos, aparece cuando se trata de la participación, pues participar es en su esencia, expresión de poder, de capacidad, de autonomía. Entonces ante esta realidad los adultos plantean que los niños, las niñas y adolescentes no están preparados, que son inmaduros, que no asumen responsabilidades, por lo tanto antes de que "participen" tiene que haber un proceso de formación para que puedan primero dialogar con los adultos y luego participar. 
En el enfoque de derechos plantea permanentemente que a participar se aprende participando. Pero esto no solo es válido para niños, niñas y adolescentes, sino también para los adultos, quienes en un importante número tampoco saben participar. Quizás saben pedir, presionar, quejarse, pero en su gran mayoría no saben participar. En algunos casos porque no existen los canales, oportunidades o facilidades para que este derecho se pueda ejercer plenamente, en otros porque no se proponen la institucionalización y democratización de las decisiones.

Es en este ámbito donde la intervención social puede jugar un rol decidor al momento de aportar en la generación de condiciones para la participación de niñas, niños y adolescentes, así como la de los adultos, y en la posibilidad de promover diálogos intergeneracionales que pongan a adultos, niños y niñas en situación de igualdad de derechos y dignidad, espacio en el cual pueden interlocutar como iguales frente a una temática que los involucra a ambos.

Lo segundo dice relación con el cambio de mentalidad o de valoración del rol de niñas y niños en la sociedad, el cual pasa necesariamente por cambios sociales y políticos que siendo importantes y necesarios no son suficientes, pues también se requieren cambios culturales, los que toman más tiempo y requieren de acciones intencionadas a nivel, nacional, local y global. Lo que queda claro desde la perspectiva de la intervención social es que esta nueva valoración se va construyendo día a día, en acciones concretas, intencionadas y con el claro objetivo de cambiar las formas de relación existentes.

La conformación de las personas en sujetos sociales, políticos, culturales, en síntesis de sujetos de derecho (personas o individuos con clara conciencia de serlo), es una construcción histórica y contextual, es decir, responde a situaciones específicas, a tiempos y espacios definidos, que no se da igual en todas la culturas, no se expresa de igual manera en los sujetos que tienen distintas biografías y experiencias diversas. El desafío consiste en que los equipos de intervención sean capaces de poner esta reflexión en los sujetos sobre los que intervienen, y esto requiere de parte de ellos una clara concepción del rol que juegan los intervenidos en el proceso que están desarrollando.

Así planteado, la valoración que se tenga de las personas afectadas por la intervención determina la forma de relación que se desarrolla con ellas. En otras palabras, esta valoración dice relación con el grado de participación que se les asigne en la transformación de sus condiciones de vida. La forma más 
común de relacionarse con los intervenidos es la de beneficiarios, es decir, una valoración pasiva del rol que cumplen en la generación de cambios. Tal como lo dice la palabra, se benefician de la acción de los interventores, en este caso, estableciéndose una forma de relación asistencial, donde se trabaja para enfrentar las situaciones coyunturales, sin buscar en conjunto con los afectados las causas que provocan esas situaciones, y por lo tanto, con ninguna posibilidad de incidir en las acciones necesarias para cambiar las condiciones de vida que les afectan negativamente.

Otra forma de valorar a los intervenidos es como usuarios, es decir, como personas que utilizan un servicio. En general las intervenciones con esta ideología a la base, entregan servicios, que corresponden a derechos, como salud, educación, protección social, asistencia en situaciones de emergencia, que al ser entregadas como "servicios", no tienen el grado de exigibilidad que todo derecho conlleva, entonces, se asume por parte de la población como un aporte que hace la autoridad o institución interventora, para mejorar su calidad de vida, pero que por ser un servicio, aunque sea fundamental, puede acabarse. Su existencia depende de la "voluntad" de la autoridad política y social, y no de una "obligación" exigible cuando se trata de un derecho humano. Esta forma de relación es evaluada a través de un formulario llamado "satisfacción del usuario".

Otra forma más reciente, es la valoración de los intervenidos como clientes, es decir, como compradores o consumidores de un servicio o un producto. Cuando el producto tiene un carácter social, puede devengar o no un costo monetario directo para el consumidor, pero se establece una relación de cliente - proveedor de bienes con quien esté entregando el producto. En la actual sociedad de consumo, donde los derechos son tratados como productos o bienes, las instituciones se ponen metas de logro; por ejemplo en los municipios y servicios públicos a las áreas que atienden público, y la satisfacción al cliente es una medida de logro. Esto está tan internalizado en la ciudadanía, que nos parece "normal" que la calidad de servicios que son derechos como la salud y la educación, esté directamente vinculada con la posibilidad del pago del cliente. Aquí la noción de derecho exigible, es cambiada por la de "derecho pagable" o "costeable".

Estas tres formas de valoración del sujeto intervenido ya sean como beneficiario, usuario o cliente, sitúa a las personas como objetos deintervención por lo tanto sin capacidad de ser parte e incidir en los diferentes momentos de la misma. La intervención es aceptada pasivamente por ellos y ellas, 
pudiendo o no producir cambios sustentables; si llegasen a ser sustentables esto sucedería de manera azarosa pues no están planificados para evaluar sustentabilidad. En general se evalúan por las metas y productos alcanzados y no por los procesos y resultados que esas acciones intencionaban. En estos tres casos la intervención social es hecha desde un enfoque de necesidades, con una visión asistencialista, donde los sujetos son pasivos, receptores de los beneficios, productos o servicios ofrecidos, donde su opinión es solicitada para definir calidad del servicio o producto y no para la determinación del resultado alcanzado, es decir del cambio logrado en ellos y ellas.

La intervención social desde el enfoque de derechos valora a los intervenidos en tanto sujetos de derechos, por lo tanto como participantes, su rol es gravitante en la consecución de la intervención. Por tanto sin su participación activa, permanente, sistemática y planificada, la intervención no logrará los resultados que se ha planteado, los que han de ser discutidos y redefinidos con los niños y niñas participantes. Aquí queda de relieve la doble, triple o múltiples funciones del equipo interventor, pues conjuntamente con promover la participación de los sujetos de intervención, tiene que formarlos para que la puedan ejercer, pero esta formación se da en la acción, es decir "a participar se aprende participando", por lo tanto, desde el inicio han de ser parte de la toma de decisiones, primero en aquellas acciones que les afecten directamente y que los tengan como protagonistas, hasta la discusión y determinación de los cambios esperados con la intervención pasando por la conversación permanente sobre la pertinencia de las distintas actividades que conlleva la intervención propuesta.

\section{Desafíos del equipo interventor}

Intervenir con enfoque de derechos requiere también de una disposición y comprensión en profundidad por parte del equipo interventor de sus implicancias. Esto quiere decir que la comprensión intelectual es insuficiente para lograr que los cambios en la situación inicial sean sustentables en el tiempo, es decir que permanezcan como capacidades de la comunidad una vez terminada la intervención, la cual siempre es finita en el tiempo. Se requiere además, una profunda convicción de que las niñas, los niños y los adolescentes son sujetos de derechos, actores sociales, que tienen la capacidad 
intrínseca para intervenir una realidad y aportar a su cambio.

Se requiere de un compromiso profesional, individual y colectivo, de que es posible lograr cambios sociales con la participación permanente y sistemática de los directamente afectados por la situación de vulneración de derechos. Compromiso profesional, porque hay que entregar lo mejor de la disciplina de la cual se es parte, para ponerla a disposición de las necesidades que irán emergiendo durante toda la intervención, la cual debe estar detalladamente planificada, permanentemente monitoreada, evaluada y sistematizada, de manera que se constituya en un aporte para la comunidad y para otras intervenciones similares. Compromiso individual, pues cada uno de los miembros del equipo establece relaciones personales e individuales con cada uno de los niños, niñas y adolescentes que están participando en la propuesta de intervención, lo que obliga a un alto grado de responsabilidad para ser coherentes entre lo que se dice y lo que se hace, donde los gestos inconscientes no traicionen el discurso de igualdad, no discriminación y respeto, que se está usando permanentemente. $\mathrm{Y}$ finalmente, compromiso colectivo, pues solo trabajando en equipo tanto entre los profesionales, como en las redes comunitarias y en las actividades con los niños, las niñas y adolescentes, será posible sustentar el cambio propuesto. El trabajo en equipo es colectivo y es el más difícil de alcanzar, toda vez que el modelo hegemónico y que permea a todos promueve el esfuerzo individual, el logro y éxito personal, desvalorizando toda acción asociativa y/o colectiva.

Estas exigencias a los equipos profesionales, así como a las instituciones que son responsables de realizar la intervención social, nos permite entrar el tercer elemento sustantivo de una intervención social con enfoque de derechos, a saber, el rol de garante de derechos y de promotores de la actoría social de niñas, niños y adolescentes participantes de la misma.

\section{Garantes de Derechos}

Los equipos de intervención así como las instituciones de las cuales forman parte, en general no saben que tienen un rol de garantes de derechos, en especial cuando están trabajando con el enfoque planteado, el que incluso pueden estar usando como fundamento teórico de su actuar. Ser garante de derechos implica asumir la responsabilidad de generar las 
condiciones de respeto, defensa y ejercicio de los derechos humanos por parte de toda persona, sin distinción alguna. El nivel de responsabilidad variará según el tipo de garante: a) ya sea garante principal, todo organismo o instancia derivada de la administración directa del Estado; b) garante co-responsable, toda organización y/o instancia de la sociedad civil; y c) garante interrelacional, que corresponde a todas las personas, la familia y la comunidad. Estas distinciones son importantes al momento de determinar las responsabilidades en la generación de condiciones y el tipo de éstas que se le exige a cada uno.

Lo primero que hay que tener claro del hecho de que los derechos humanos sean exigibles, es el ante quién o qué ente son exigibles, pues éste tendrá la obligación de responder ante esta exigibilidad.

Lo segundo, es que esta exigibilidad se reclama fundamentalmente ante el Estado, quien es el garante principal de derechos, por lo cual tiene la obligación, legalmente establecida por la firma de los instrumentos internacionales de derechos humanos, de generar las condiciones políticas, económicas, culturales y sociales; jurídicas, legales, legislativas y de infraestructura para que los derechos puedan ser ejercidos por todos los y las habitantes del país. Es el Estado quien debe responder por las violaciones o vulneraciones de los derechos humanos ante la ciudadanía y ante los organismos internacionales de Naciones Unidas.

En relación a los derechos del niño, se amplía y distribuye esta responsabilidad a la sociedad civil, la familia y la comunidad, por el particular período de crecimiento en que están los niños y las niñas. Esto no minimiza en nada la responsabilidad del Estado, pero se amplia a las otras instancias por el cambio cultural que implica el poner en práctica la Convención sobre los Derechos del Niño, por una parte, y por la influencia que en el desarrollo de niñas y niños tienen las diferentes expresiones organizadas que se da la sociedad, por otra.

Volviendo a la intervención social con enfoque de derechos y su rol de garante de derechos, este tiene dos niveles distintos pero complementarios. Uno, el que está directamente vinculado con la intervención y el vínculo que se establece con los participantes de ella. A saber, el ser garante coresponsable (si es un organización de la sociedad civil) implica que en la intervención se asegure una planificación en la que la participación de niñas, niños y adolescentes está presente, en todos los aspectos de ejecución de la misma donde ellas y ellos puedan ser parte y aportar, como fue explicitado 
anteriormentecuando hablamos dela valoración del sujeto intervenido. Ahora, en este nivel, siempre está presente el que cada miembro del equipo, cada persona es garante interrelacional, es decir, responsable de que las relaciones que se dan entre ellos y los niños, niñas y adolescentes son respetuosas de sus derechos, su dignidad, no discriminatorias, promotoras del desarrollo pleno de todas sus capacidades, etc.

El otro nivel está vinculado con la forma en que se relaciona el equipo interventor en tanto instancia, con el entorno organizacional, institucional y social donde realiza la intervención. El ser garante co - responsable, implica que la institución y todos sus miembros tienen que buscar formas de aportar a la generación de condiciones de respeto de los derechos en los niveles en los que está trabajando, donde se asumen responsabilidades colectivas en pos del bien común, del mejoramiento de las condiciones de vida de niñas y niños, etc., en otras palabras, han de trabajar en red, promover la asociatividad, la responsabilización, la generación de propuestas por parte de la comunidad y de sus organizaciones que incluyan a niñas, niños y adolescentes. Por otra parte, en este nivel, a la instancia u organización interviniente le cabe también una responsabilidad de formación de garantes.

Una intervención social con enfoque de derechos, tiene que establecer al momento de hacer la evaluación de la situación inicial, quiénes son los garantes de derechos presentes en ese espacio-tiempo determinado en que se hará la intervención, y qué grado de comprensión de su responsabilidad de garantes tienen. Esto determina las actividades que se realizarán dada esa situación inicial, las que han de ser monitoreadas, evaluadas y sistematizadas al igual que el resto de las acciones.

En el mismo proceso de asunción del rol de garante de derechos en tanto instancia que ejecuta una intervención social y como equipo profesional, se debe asumir el de promotor de la actoría social de niñas, niños y adolescentes. Esto no es muy diferente a lo que ya se explicitó acerca de la participación real de ellos y ellas en los procesos de intervención, si no más bien se acentúa el carácter político y cultural de este rol. Político, en tanto niñas y niños son personas con sus propios derechos, con habilidades específicas que están a disposición y deben ser respetadas por los adultos y reconocidas por la comunidad, a la vez que explicitar que el ser sujeto de derechos es una posición de poder el cual otorga a las personas la capacidad para dirigir libremente su vida sin estar a merced de otros, lo que no implica claudicar la responsabilidad de los adultos de proteger a la infancia y la 
niñez. En el proceso de intervención estas características propias han de ser promovidas y a la vez ejercidas por niñas y niños y reconocidas por los adultos, acorde a las responsabilidades de ambas partes. Cultural, pues el proceso de cambio intencionado de la intervención pone de relieve las necesarias transformaciones de las relaciones entre niños, niñas y adultos, rompiendo la matriz de sujeción existente, visibilizando su participación en la generación de propuestas hacia la comunidad, relevando el rol que tienen en la vida de la comunidad. A través de esto la intervención social con enfoque de derechos aporta al proceso de cambio de la matriz cultural dominante, que pone a niñas, niños y adolescentes como seres inferiores, incompletos, inmaduros, resituándolos como agentes de cambio social, como actores conscientes de la realidad que viven y a la cual pueden y deben aportar sus visiones de mejoramiento. 


\section{A modo de conclusión}

Para alcanzar una intervención social que tenga en su base el enfoque de derechos, es necesario tener presente, que estamos hablando de aportar a un proceso que ya está andando y que seguirá una vez hayamos terminado la intervención específica que se realice. En otras palabras, estamos siendo parte de un continuo al cual nos introducimos de manera consciente y con objetivos de cambio claros y específicos a la realidad que se interviene.

Tener claridad que la situación política y social actual puede ser caracterizada como una en que una de las problemáticas centrales es la de la exclusión en sus diversos planos, que se expresa en fragmentación social; en la ruptura de las solidaridades; en la fractura de las redes de contención y apoyo; y en la focalización de muchas de las intervenciones venidas desde el Estado con el consiguiente abandono de la universalidad de los derechos humanos.

Tener claridad del horizonte de intervención ${ }^{[19]}$, o de la teoría de cambio a la base de la misma, hace la diferencia entre estar trabajando con y desde el enfoque de derechos, o solo administrando el modelo y aportando a la contención de la crisis social que esta situación puede producir.

El sentido de la intervención es, entonces, la integración social de acuerdo al reconocimiento, respeto y ejercicio de los derechos por parte de la ciudadanía y en particular de niñas, niños y adolescentes; la reconstrucción de las solidaridades entendida ésta como el soporte del tejido de redes y el intercambio de capacidades entre diferentes grupos sociales. En otras palabras, se trata de lograr procesos crecientes de integración, cohesión e inclusión social; y la visibilización y relevamiento de las capacidades de niñas, niños y adolescentes en tanto actores sociales con propuestas de cambio social viables y sustentables.

Desde una perspectiva más técnica, tener claridad de la teoría de cambio que guía la intervención social con enfoque de derechos, permite relevar los resultados y procesos por sobre los productos y las metas; establece lenguajes y principios de acción comunes y conocidos por todos los

[19] Carballeda, op. Cit. 2005. 
que son parte de la intervención, tanto del equipo como de los participantes; identifica los recursos y su adecuación al propósito; permite diseñar planes de acción realistas; establece y clarifica las líneas de responsabilidad internas y externas; permite y facilita las realización de evaluaciones significativas y de sistematización de las experiencias. 


\section{Bibliografía}

- Carballeda J. Alfredo, 2005, La intervención en lo social. Exclusión e integración en los nuevos escenarios sociales, Editorial Paidós, Serie Tramas Sociales, Buenos Aires, Argentina.

- Convención sobre los Derechos del Niño, 1989, ONU.

- DeclaracióndelMilenioyObjetivosdeDesarrollo,2000,www.developmentgoal. org

- Declaración Universal de los Derechos Humanos, 1948, ONU.

- De Oliveira Francisco, 2005, "Sobre las posibilidades del desarrollo", En: América Latina 2004-2005. Democracia y Desarrollo: Una mirada desde la sociedad civil, Informe de ALOP.

- Domínguez V. Andrés, 1988, El Poder y los Derechos Humanos, Centro de Estudios Políticos latinoamericanos, Simón Bolívar, Terranova Editores, Santiago, Chile.

- Gaitán Lourdes, 2006, Sociología de la Infancia, Nuevas Perspectivas, Editorial Síntesis, Madrid, España

- Liebel, Manfred, 1994, Protagonismo Infantil, Movimientos de niños trabajadores en América Latina, Editorial Nueva Nicaragua, Nicaragua.

- Save the Children, 2005, Programación de los Derechos del Niño. Cómo aplicar un Enfoque de Derechos en la programación. Un manual para los miembros de la Alianza Internacional Save the Children. Segunda Edición. Lima-Perú.

- Valverde Mosquera Francis, 2004, Apuntes sobre enfoque de derechos, ACHNU, sin publicar.

- Valverde Francis y Latorre Soledad, 2007, Presentación Módulo Intervención Social, Diplomado Niñez y Políticas Publicas, Universidad de Chile, Santiago, Chile, sin publicar.

- Sen Amartya, 2000, Desarrollo y Libertad; Editorial Planeta, Barcelona, España.

- Touraine, Alain, 1994, Crítica de la Modernidad, Fondo de Cultura Económica de Argentina, Buenos Aires, Argentina.

. 2006, Un nuevo paradigma para comprender el mundo de hoy, Editorial Paidós, Buenos Aires, Argentina. 